\title{
The potential roles of stem cell-derived extracellular vesicles as a therapeutic tool
}

\author{
Yu-Chin Huang ${ }^{1,2}$, Liang-Chuan Lai ${ }^{1,3}$ \\ ${ }^{1}$ Graduate Institute of Physiology, College of Medicine, National Taiwan University, Taipei; ${ }^{2}$ Taoyuan General Hospital, Ministry of Health and \\ Welfare, Taoyuan City; ${ }^{3}$ Bioinformatics and Biostatistics Core, Center of Genomic and Precision Medicine, National Taiwan University, Taipei \\ Contributions: (I) Conception and design: All authors; (II) Administrative support: LC Lai; (III) Provision of study materials or patients: YC Huang; \\ (IV) Collection and assembly of data: YC Huang; (V) Data analysis and interpretation: YC Huang; (VI) Manuscript writing: All authors; (VII) Final \\ approval of manuscript: All authors. \\ Correspondence to: Liang-Chuan Lai. Graduate Institute of Physiology, College of Medicine, National Taiwan University, Taipei 110. Email: llai@ntu.edu.tw.
}

\begin{abstract}
Extracellular vesicles (EVs) of mesenchymal stem cells (MSCs) are secreted by live cells and possess the same regenerative potential and immunomodulatory ability as their parental cells. Clinical applications of MSC-EVs could overcome the shortage of MSCs for treatment of cancer and other diseases and impact the field of regenerative medicine from cellular to acellular therapy. For use of MSC-EVs as a clinical agent, various engineered $\mathrm{EVs}$ have been manufactured and their therapeutic effects on various diseases demonstrated in preclinical studies and clinical trials. However, MSC-EVs are heterogeneous, and many of their characteristics are still unknown. Many barriers still need to be surmounted before MSC-EVs can be used as biomedical agents.
\end{abstract}

Keywords: Mesenchymal stem cell (MSC); extracellular vesicle (EV); therapeutic tool

Submitted Oct 04, 2019. Accepted for publication Nov 10, 2019.

doi: 10.21037/atm.2019.11.66

View this article at: http://dx.doi.org/10.21037/atm.2019.11.66

\section{Introduction}

Extracellular vesicles (EV), round nanosized particles surrounded by a bilayer-lipid membrane, were first discovered in 1983 (1). Almost all kinds of prokaryotes and eukaryotes have the ability to secrete, EVs indicating some important roles in multifaceted physiological and pathological functions of intercellular communication (2-5). Mesenchymal stem cells (MSCs) are the most widely used somatic stem cells in regenerative medicine and exhibit potential therapeutic effects in many preclinical disease models because of their differentiation ability and paracrine effects (6-9). Furthermore, MSC-EVs exhibit the biological properties of their parent cells (10) and have been successfully applied for treating various diseases (9). As compared with MSC therapy, application of MSC$\mathrm{EVs}$ is simpler because of easier manipulation and storage. Various engineered MSC-EVs extend the medical scope of application by providing precise and targeted clinical therapy. However, although they have a promising role in regenerative medicine, MSC-EVs are heterogeneous, with great variety of compositions, and are affected by different factors during their production and use $(11,12)$. Therefore, there are still some hurdles to be overcome before complete translation to clinical practice. In this editorial review, we focus on the potential applications of MSC-EV-based therapeutics from bench to bedside.

\section{EVs}

EVs are nanosized particles with a lipid membrane, enclosed by and released from a majority of cell types, and contain proteins, lipids, and genetic material. These secreted particles have the ability to transmit bioactive components between cells, activating signaling pathways and facilitating intercellular communication $(2-5,13)$. The main types of EVs include the following: exosomes (diameter range 30-150 nm), emitted from 
intracellular endosomes (14); microvesicles (diameter range $50-1,000 \mathrm{~nm}$ ), originating from outward invaginations of plasma membrane regions $(11,15)$; and apoptotic bodies (diameter range 50-5,000 nm), originating as fragments of cells undergoing programmed death (16). Both exosomes and microvesicles contain proteins derived from their parent cells, but exhibit different properties because of their differing mechanisms of biogenesis $(17,18)$. Exosomes carry lipid molecules from both the Golgi apparatus and the plasma membrane, whereas microvesicles carry lipids only from the plasma membrane (19). In the field of EV research, the nomenclature is somewhat chaotic due to overlapping characterizations of different subtypes of exosomes and microvesicles, in part because the physical separation of EVs by particle size and the discrimination between markers of different pathways of biogenesis is impracticable (20). According to the Minimal Information for Studies of Extracellular Vesicles (MISEV) 2018 guidelines, unless authors can establish specific markers of subcellular origin, it is advisable to use of operational terms for EVs, with a description of physical characteristics, such as size, biochemical composition, conditions, or cell of origin, to distinguish subsets of EVs $(18,21,22)$.

\section{From cellular to acellular therapy in regenerative medicine}

MSCs represent a promising stem cell population for applications in regenerative medicine. Plenty of evidence has documented that MSCs, based on their differentiation potential, possess therapeutic effects in preclinical models of many immunological and degenerative diseases (6-8). However, studies of cell tracking after MSCs were transplanted revealed that most MSCs did not engraft into the injured tissue or differentiate into functional cells $(23,24)$. In fact, most MSCs were trapped in liver, spleen, and lungs, with $<1 \%$ of them migrating to the target tissue (25). Therefore, the effect of MSCs was proposed to be due to the secretion of paracrine factors rather than differentiation ability $(26,27)$. Thus, the intercellular communication between MSCs and tissue cells gradually became the focus in regenerative medicine.

Haynesworth et al. were the first to prove that MSCs synthesize and secrete an extensive variety of growth factors, chemokines, and cytokines that could exert significant effects on nearby and distant cells (28). Most investigations also showed that stem cell-conditioned media exhibit the biological properties of parent cells (10), possess the ability to promote neovascularization, and ameliorate ischemic renal and limb tissue injury by increasing angiogenesis (29-31). Thereafter, the therapeutic potential of the MSC secretome in EVs, including both soluble and insoluble factors, was analyzed and investigated $(32,33)$. In recent publications, MSC-EVs were used successfully to repair injured cell, enhance tissue regeneration, and control overt inflammation (9).

MSC cell therapy has many drawbacks due to the use of living cells. For example, the biological potency of the viable replicating cells cannot be "turned off," even when the therapy has been terminated (34), and the possibility of englutted MSCs cannot be eliminated $(35,36)$. During transplantation of MSCs by intravascular infusion, because of the large cell size, the distal microvasculature may be occlusive $(37,38)$. Moreover, promotion of tumor growth is another important consideration when utilizing human MSCs as therapeutic agents (39). Some animal studies reported that tissue ossification or calcification, resulting from multipotent MSCs differentiating into osteocytes and chondrocytes, is a cause for long-term safety concerns (40). In contrast, nanosized EVs minimize the risk of occlusion, avoiding potential harmful side effects of cells by decaying spontaneously over time. MSC-EVs have no risk of tumorigenicity or extraosseous calcification. Furthermore, MSC-EVs have the advantage of a lower possibility of immune rejection following in vivo allogeneic administration (41). These advances represent a new era of "acellular" therapy, which can overcome the unpredictable effects of viable cells in biotherapy and facilitate the same therapeutic applications (12).

\section{The potential application of MSC-EV-based therapeutics from bench to bedside}

EV-based therapeutics have been applied to the treatment of various organ diseases in human preclinical studies, but the efficacy has been controversial. On the other hand, many clinical trials have been conducted using EVs modified by genetic engineering, which will extend the scope of biomedical applications and provide a powerful tool to solve clinical problems.

\section{Application of MSC-EVs in drug delivery}

MSC-EVs are endogenous vectors, shuttling between cells with excellent biocompatibility. This natural "truck" can transport various bioactive components, such as small 
molecules and drugs. Yet, the great challenge is how to load the cargo into MSC-EVs. Previously, cells meant for therapeutic applications were manipulated by genetic technology to overexpress desired proteins or RNAs, but with MSC-EVs, cargo has been packed into MSCEVS by way of co-incubation (42), electroporation (43), or sonication (44). In addition, to reach their destination correctly, EVs express targeting peptides on their surface, encoded by genetically modified parent cells. The targeting peptides of EVs can facilitate fusion with the membrane of target cells (43). This approach can be applied to avoid the side effects of chemotherapy drugs. In other words, drug particles can be delivered into MSC-EVs that display peptides targeted against tumors. So not only do MSC-EVs reach the correct destination, they also elevate antitumor effects.

\section{Application of MSC-EVs in oncology}

MSC-EVs demonstrated great promise in treating tumors, but controversial results still remain. For example, like MSCs, secreted MSC-EVs possess immunomodulatory ability. Dendritic cell (DC)-derived MSC-EVs were predicted to activate patients' immune response to eliminate cancer cells. In some preclinical trails, the autologous DC-derived MSC-EVs trained by the tumor antigenic peptides in vitro did demonstrate antitumor ability $(45,46)$, but subsequent studies conducted in 2005 revealed little therapeutic effect (47-49). Therefore, more experiments are warranted to optimize the antitumor applications of MSC-EVs.

\section{Application of MSC-EVs in hereditary disease}

As a natural delivery tool, EVs could potentially remedy mutations related to hereditary diseases by merging with recipient cells and transferring biomaterials, including RNA, miRNA, protein, and even DNA. In this way, marked MSC-EVs binding to targeted cells could mediate the exchange of genetic information and signal transduction. In recent publications, researchers have successfully treated various inherited genetic diseases using therapeutic MSC-EVs (50-52).

\section{MSC-EVs modified by engineered biotechnology}

For better therapeutic efficiency, modification of the surface molecules on MSC-EVs has been developed to increase retention in the bloodstream. By altering features such as particle size, surface receptors, or membrane charge distribution, MSC-EVs can avoid being eliminated by the liver, kidney, and/or reticuloendothelial system (52-55).

Targeted therapy could be achieved by engineering the targeting peptides on the MSC-EV surface to home to specific tissues or cells, and this process would promote more accurate personalized medicine (56,57). In 2018, a set of EXOsomal transfer into cells (EXOtic) devices was designed to produce customized exosomes by engineering mammalian cells with three candidate genes related to exosome biogenesis, which resulted in a more than 15 -fold increase in the output of exosomes. RNA delivery-based therapeutic applications would be made more convenient by using such devices (58). Moreover, Votteler et al. designed self-assembling 'enveloped protein nanocages' (EPNs). Robust EPN biogenesis required protein sequence elements that encode three distinct functions: membrane binding, self-assembly, and recruitment of the endosomal sorting complexes, which were required for efficiently transferring cargo to the cytoplasm of recipient cells (59).

Researchers have also developed bioinspired exosomemimetic nanovesicles for delivering chemotherapeutics to tumor tissue after systemic administration. The exosomemimetic nanovesicles are produced by breakdown of monocytes or macrophages using serial extrusion through filters with diminishing pore sizes $(10,5$, and $1 \mu \mathrm{m})$. These cell-derived nanovesicles have similar characteristics to the exosomes, but have 100-fold higher yield (60).

A novel streamlined microfluidic cell culture platform, called a PDMS microfluidic cell culture chip, which integrates harvesting, antigenic modification, and photorelease of surface engineered exosomes in one workflow, was created in 2019 (61). Researchers claimed that the PDMS microfluidic cell culture chip could easily harvest intact, engineered antigenic exosomes which can be employed in activating antitumor responses.

Together, the progress in engineered MSC-EVs has expedited the translation of stem cell-derived EVs into clinical applications.

\section{Conclusions and future perspectives}

Despite the potential of MSC-EVs to be applied in various clinical settings, as mentioned above, there are some hurdles that must be overcome. First, due to the lack of standardization in exosome isolation and analytic methods, direct application of MSC-EVs is somewhat restricted. 
Second, quality control (e.g., identity, purity, potency, and stability) and pharmacodynamics (e.g., mode of action, active ingredient) are things that must be clarified before a clinical trial is undertaken. Third, MSC-EV populations are heterogeneous, with greatly varying compositions (11), as a result of various factors during processing (12), which could explain the unpredictable therapeutic efficacy observed in some clinical trials. To address this issue, most MSC-EV preparations are characterized according to the MISEV2014 guidelines, which recommend specific criteria for definition and classification of EVs (62). Recently, MISEV2014 has been updated and extended as MISEV2018, which includes specific standard criteria for MSC-EVs to accelerate the field toward clinical applications (22). Lastly, for safety considerations, the usage of EVs, under the umbrella of "biological therapeutics," should be defined by a precise set of regulatory requirements $(63,64)$, follow internationally harmonized regulatory frameworks, and meet Good Manufacturing Practice standards (65).

From cell therapy to acellular therapy, the main goal of MSC-EVs is to regenerate or repair injured organs and cells. Nanosized MSC-EVs may resolve a bottleneck of current medicine by rejuvenating and revitalizing cells and tissues, and provide precise and targeted medical treatment.

\section{Acknowledgments}

We thank Melissa Stauffer for editorial assistance.

\section{Footnote}

Conflicts of Interest: The authors have no conflicts of interest to declare.

Ethical Statement: The authors are accountable for all aspects of the work in ensuring that questions related to the accuracy or integrity of any part of the work are appropriately investigated and resolved.

\section{References}

1. Harding CV, Heuser JE, Stahl PD. Exosomes: looking back three decades and into the future. J Cell Biol 2013;200:367-71.

2. Théry C, Zitvogel L, Amigorena S. Exosomes: composition, biogenesis and function. Nat Rev Immunol 2002;2:569-79.

3. Xu R, Rai A, Chen M, et al. Extracellular vesicles in cancer
- implications for future improvements in cancer care. Nat Rev Clin Oncol 2018;15:617-38.

4. Pi F, Binzel DW, Lee TJ, et al. Nanoparticle orientation to control RNA loading and ligand display on extracellular vesicles for cancer regression. Nat Nanotechnol 2018;13:82-9.

5. Théry C. Cancer: Diagnosis by extracellular vesicles. Nature 2015;523:161-2.

6. Bateman ME, Strong AL, Gimble JM, et al. Concise Review: Using Fat to Fight Disease: A Systematic Review of Nonhomologous Adipose-Derived Stromal/Stem Cell Therapies. Stem Cells 2018;36:1311-28.

7. Borakati A, Reza M, Pouya M, et al. A Systematic Review And Meta-Analysis of Clinical Trials of Mesenchymal Stem Cell Therapy for Cartilage Repair. Curr Stem Cell Res Ther 2018;13:215-25.

8. Jeong H, Yim HW, Park HJ, et al. Mesenchymal Stem Cell Therapy for Ischemic Heart Disease: Systematic Review and Meta-analysis. Int J Stem Cells 2018;11:1-12.

9. Bjørge IM, Kim SY, Mano JF, et al. Extracellular vesicles, exosomes and shedding vesicles in regenerative medicine - a new paradigm for tissue repair. Biomater Sci 2017;6:60-78.

10. Takahashi M, Li TS, Suzuki R, et al. Cytokines produced by bone marrow cells can contribute to functional improvement of the infarcted heart by protecting cardiomyocytes from ischemic injury. Am J Physiol Heart Circ Physiol 2006;291:H886-93.

11. György B, Szabo TG, Pasztoi M, et al. Membrane vesicles, current state-of-the-art: emerging role of extracellular vesicles. Cell Mol Life Sci 2011;68:2667-88.

12. Reiner AT, Witwer KW, van Balkom BWM, et al. Concise Review: Developing Best-Practice Models for the Therapeutic Use of Extracellular Vesicles. Stem Cells Transl Med 2017;6:1730-9.

13. Barile L, Moccetti T, Marbán E, et al. Roles of exosomes in cardioprotection. Eur Heart J 2017;38:1372-9.

14. Corrado C, Raimondo S, Chiesi A, et al. Exosomes as intercellular signaling organelles involved in health and disease: basic science and clinical applications. Int J Mol Sci 2013;14:5338-66.

15. Riazifar M, Pone EJ, Lotvall J, et al. Stem Cell Extracellular Vesicles: Extended Messages of Regeneration. Annu Rev Pharmacol Toxicol 2017;57:125-54.

16. Hauser P, Wang S, Didenko VV. Apoptotic Bodies: Selective Detection in Extracellular Vesicles. Methods Mol Biol 2017;1554:193-200.

17. Luan X, Sansanaphongpricha K, Myers I, et al. 
Engineering exosomes as refined biological nanoplatforms for drug delivery. Acta Pharmacol Sin 2017;38:754-63.

18. Raposo G, Stoorvogel W. Extracellular vesicles: exosomes, microvesicles, and friends. J Cell Biol 2013;200:373-83.

19. Barile L, Vassalli G. Exosomes: Therapy delivery tools and biomarkers of diseases. Pharmacol Ther 2017;174:63-78.

20. Cocucci E, Meldolesi J. Ectosomes and exosomes: shedding the confusion between extracellular vesicles. Trends Cell Biol 2015;25:364-72.

21. Witwer KW, Van Balkom BWM, Bruno S, et al. Defining mesenchymal stromal cell (MSC)-derived small extracellular vesicles for therapeutic applications. J Extracell Vesicles 2019;8:1609206.

22. Théry C, Witwer KW, Aikawa E, et al. Minimal information for studies of extracellular vesicles 2018 (MISEV2018): a position statement of the International Society for Extracellular Vesicles and update of the MISEV2014 guidelines. J Extracell Vesicles 2018;7:1535750.

23. Duffield JS, Park KM, Hsiao LL, et al. Restoration of tubular epithelial cells during repair of the postischemic kidney occurs independently of bone marrow-derived stem cells. J Clin Invest 2005;115:1743-55.

24. Biancone L, Bruno S, Deregibus MC, et al. Therapeutic potential of mesenchymal stem cell-derived microvesicles. Nephrol Dial Transplant 2012;27:3037-42.

25. Phinney DG, Prockop DJ. Concise Review: Mesenchymal Stem/Multipotent Stromal Cells: The State of Transdifferentiation and Modes of Tissue Repair-Current Views. Stem Cells 2007;25:2896-902.

26. Baglio SR, Pegtel D, Baldini N. Mesenchymal stem cell secreted vesicles provide novel opportunities in (stem) cellfree therapy. Front Physiol 2012;3:359.

27. Lai RC, Yeo RW, Lim SK. Mesenchymal stem cell exosomes. Semin Cell Dev Biol 2015;40:82-8.

28. Haynesworth SE, Baber MA, Caplan AI. Cytokine expression by human marrow-derived mesenchymal progenitor cells in vitro: effects of dexamethasone and IL-1 alpha. J Cell Physiol 1996;166:585-92.

29. Kinnaird T, Stabile E, Burnett MS, et al. Bone marrowderived cells for enhancing collateral development: Mechanisms, animal data, and initial clinical experiences. Circ Res 2004;95:354-63.

30. Min JY, Sullivan MF, Yang Y, et al. Significant improvement of heart function by cotransplantation of human mesenchymal stem cells and fetal cardiomyocytes in postinfarcted pigs. Ann Thorac Surg 2002;74:1568-75.

31. Kinnaird T, Stabile E, Burnett MS, et al. Marrow-Derived
Stromal Cells Express Genes Encoding a Broad Spectrum of Arteriogenic Cytokines and Promote In Vitro and In Vivo Arteriogenesis Through Paracrine Mechanisms. Circ Res 2004;94:678-85.

32. Yu B, Zhang X, Li X. Exosomes derived from mesenchymal stem cells. Int J Mol Sci 2014;15:4142-57.

33. Lavoie JR, Rosu-Myles M. Uncovering the secretes of mesenchymal stem cells. Biochimie 2013;95:2212-21.

34. Lai RC, Chen TS, Lim SK. Mesenchymal stem cell exosome: a novel stem cell-based therapy for cardiovascular disease. Regen Med 2011;6:481-92.

35. Freeman BT, Kouris NA, Ogle BM. Tracking Fusion of Human Mesenchymal Stem Cells After Transplantation to the Heart. Stem Cells Transl Med 2015;4:685-94.

36. Vassilopoulos G, Wang PR, Russell DW. Transplanted bone marrow regenerates liver by cell fusion. Nature 2003;422:901-4.

37. Poh K-K, Sperry E, Young RG, et al. Repeated direct endomyocardial transplantation of allogeneic mesenchymal stem cells: Safety of a high dose, "off-the-shelf", cellular cardiomyoplasty strategy. Int J Cardiol 2007;117:360-4.

38. Furlani D, Ugurlucan M, Ong L, et al. Is the intravascular administration of mesenchymal stem cells safe? Mesenchymal stem cells and intravital microscopy. Microvasc Res 2009;77:370-6.

39. Zhu W, Huang L, Li Y, et al. Mesenchymal stem cellsecreted soluble signaling molecules potentiate tumor growth. Cell Cycle 2011;10:3198-207.

40. Breitbach M, Bostani T, Roell W, et al. Potential risks of bone marrow cell transplantation into infarcted hearts. Blood 2007;110:1362-9.

41. Stoltz JF, de Isla N, Li YP, et al. Stem Cells and Regenerative Medicine: Myth or Reality of the 21th Century. Stem Cells Int 2015;2015:734731.

42. Didiot MC, Hall LM, Coles AH, et al. Exosome-mediated Delivery of Hydrophobically Modified siRNA for Huntingtin mRNA Silencing. Mol Ther 2016;24:1836-47.

43. Alvarez-Erviti L, Seow Y, Yin H, et al. Delivery of siRNA to the mouse brain by systemic injection of targeted exosomes. Nat Biotechnol 2011;29:341-5.

44. Lamichhane TN, Jeyaram A, Patel DB, et al. Oncogene Knockdown via Active Loading of Small RNAs into Extracellular Vesicles by Sonication. Cell Mol Bioeng 2016;9:315-24.

45. Zitvogel L, Regnault A, Lozier A, et al. Eradication of established murine tumors using a novel cell-free vaccine: dendritic cell-derived exosomes. Nat Med 1998;4:594-600.

46. Chaput N, Schartz NE, Andre F, et al. Exosomes as potent 
cell-free peptide-based vaccine. II. Exosomes in CpG adjuvants efficiently prime naive Tc1 lymphocytes leading to tumor rejection. J Immunol 2004;172:2137-46.

47. Morse MA, Garst J, Osada T, et al. A phase I study of dexosome immunotherapy in patients with advanced nonsmall cell lung cancer. J Transl Med 2005;3:9.

48. Robbins PD, Morelli AE. Regulation of immune responses by extracellular vesicles. Nat Rev Immunol 2014;14:195-208.

49. Besse B, Charrier M, Lapierre V, et al. Dendritic cellderived exosomes as maintenance immunotherapy after first line chemotherapy in NSCLC. Oncoimmunology 2015;5:e1071008.

50. A novel gene containing a trinucleotide repeat that is expanded and unstable on Huntington's disease chromosomes. The Huntington's Disease Collaborative Research Group. Cell 1993;72:971-83.

51. Hosseini-Beheshti E, Grau GER. Extracellular vesicles as mediators of immunopathology in infectious diseases. Immunol Cell Biol 2018. [Epub ahead of print].

52. Romagnoli GG, Zelante BB, Toniolo PA, et al. Dendritic Cell-Derived Exosomes may be a Tool for Cancer Immunotherapy by Converting Tumor Cells into Immunogenic Targets. Front Immunol 2015;5:692.

53. Fuhrmann G, Neuer AL, Herrmann IK. Extracellular vesicles - A promising avenue for the detection and treatment of infectious diseases? Eur J Pharm Biopharm 2017;118:56-61.

54. Fatima F, Nawaz M. Stem cell-derived exosomes: roles in stromal remodeling, tumor progression, and cancer immunotherapy. Chin J Cancer 2015;34:541-53.

55. Hwang I. Cell-cell communication via extracellular membrane vesicles and its role in the immune response. Mol Cells 2013;36:105-11.

56. Lu M, Xing H, Xun Z, et al. Functionalized extracellular vesicles as advanced therapeutic nanodelivery systems. Eur J Pharm Sci 2018;121:34-46.

Cite this article as: Huang YC, Lai LC. The potential roles of stem cell-derived extracellular vesicles as a therapeutic tool. Ann Transl Med 2019;7(22):693. doi: 10.21037/atm.2019.11.66
57. Irimie AI, Sonea L, Jurj A, et al. Future trends and emerging issues for nanodelivery systems in oral and oropharyngeal cancer. Int J Nanomedicine 2017;12:4593606.

58. Kojima R, Bojar D, Rizzi G, et al. Designer exosomes produced by implanted cells intracerebrally deliver therapeutic cargo for Parkinson's disease treatment. Nat Commun 2018;9:1305.

59. Votteler J, Ogohara C, Yi S, et al. Designed proteins induce the formation of nanocage-containing extracellular vesicles. Nature 2016;540:292-5.

60. Jang SC, Kim OY, Yoon CM, et al. Bioinspired exosome-mimetic nanovesicles for targeted delivery of chemotherapeutics to malignant tumors. ACS Nano 2013;7:7698-710.

61. Zhao Z, McGill J, Gamero-Kubota P, et al. Microfluidic on-demand engineering of exosomes towards cancer immunotherapy. Lab Chip 2019;19:1877-86.

62. Lötvall J, Hill AF, Hochberg F, et al. Minimal experimental requirements for definition of extracellular vesicles and their functions: a position statement from the International Society for Extracellular Vesicles. J Extracell Vesicles 2014;3:26913.

63. Gimona M, Pachler K, Laner-Plamberger S, et al. Manufacturing of Human Extracellular Vesicle-Based Therapeutics for Clinical Use. Int J Mol Sci 2017;18. doi: http://dx.doi.org/10.3390/ijms18061190.

64. Ilic N, Savic S, Siegel E, et al. Examination of the regulatory frameworks applicable to biologic drugs (including stem cells and their progeny) in Europe, the U.S., and Australia: part I--a method of manual documentary analysis. Stem Cells Transl Med 2012;1:898-908.

65. Lener T, Gimona M, Aigner L, et al. Applying extracellular vesicles based therapeutics in clinical trials an ISEV position paper. J Extracell Vesicles 2015;4:30087. 